\title{
Elastic and effective cloud-based solution in online education
}

\author{
Yi Jin ${ }^{1,}$, Jianhua Huang ${ }^{1, b}$, Cheng $\mathrm{Li}^{1, \mathrm{c}}$, Qi Ye ${ }^{1, \mathrm{~d}}$, Jun Yao ${ }^{1, \mathrm{e}}$ \\ ${ }^{1}$ East China University of Science and Technology, Shanghai, China \\ ajinyi@ecust.edu.cn, bjhhuang@ecust.edu.cn, '045130148@mail.ecust.edu.cn, dyeh_qi1125@ecus \\ t.edu.cn, èaojun@ecust.edu.cn
}

Keywords: cloud, Scaling, OpenStack, education, scheduling

\begin{abstract}
By default, OpenStack scheduling policy is random scheduling, without considering load balancing of the resource pool. This paper proposes an elastic and effective cloud-based solution in online education. OpenStack integrating with Cloudify is used to build the cloud. An elastic scaling approach of resource provisioning is described in order to guarantee the performance of applications while the load fluctuations of online education applications occur. The solution improves the performance of applications, and adapts to the demand for resource provisioning in a private cloud. Experimental evaluation demonstrates the effectiveness of the proposed online education cloud.
\end{abstract}

\section{Introduction}

The emerging of online education technologies enables us to get the new higher education opportunity through the Internet. In the pattern students register to learn a variety of video courses through the Internet, and finally get their degrees. However, with the expansion of students, this pattern is still facing several challenges. For examples, sometimes sharp fluctuations in server load (such as course selection) require more computing resources, loads of some servers are very heavy and others may be much low. Cloud computing is a new kind of shared infrastructure, which provides users with enough flexibility for dynamic allocation of computing resources, improves the utilization of hardware resources, and effectively reduces IT costs [1]. The benefits of cloud computing let some online education institutions transfer their E-learning applications towards cloud platforms. However, migrating the applications to the clouds is not an easy process. User access characteristics in online education have not yet been taken into account in cloud platforms. It is not easy for an education cloud to deal with the demand for resources caused by the access of a massive number of students, especially in the limited resources.

This paper presents an elastic and effective cloud-based solution in order to solve the above problems. We discuss a cloud architecture built by OpenStack [2] for online education. In order to guarantee the performance of applications and recycle excess resources, a cloud middleware called Cloudify is integrated with OpenStack to expand or shrink resource provisioning dynamically according to the workload of applications.

\section{Related work}

Compared with traditional technologies, cloud computing provides users with the flexibility of sharing IT infrastructure and provisioning on-demand resources. There are a lot of online education applications to begin to turn towards cloud computing. One of the earlier projects where the cloud is used in education is StarHPC, which is a software tool for academic research [3]. This project allows students to use Amazon's EC2 resources in their laboratory. Cisco recently migrated its learning environment providing students with theoretical background and practical experience on its cloud platform [4]. Zhao Shaka [5] designed an education cloud architecture based on OpenStack. A strategy based on task scheduling and load balancing was proposed in order to balance server's resource load on the basis of ensuring its service performance and execution efficiency. ECAP [6] is a kind of distributed self-elasticity architecture based on cloud computing. The architecture built an 
IaaS platform combining Cloudify PaaS platform for expanding and contracting of services. Li Jing [7] designed and implemented a distance education resource sharing system based on cloud computing, in which the scheduling policy is optimized. Ma Zhichao[8] proposed a scheduling algorithm to resolve the shortage of scheduling algorithm in OpenStack in order to improve the effect and energy-saving of resources.

There are still some problems to be solved in online education, such as the issue of service performance caused by load fluctuations. In this paper, we propose a cloud-based solution in online education. The open source project OpenStack integrating with Cloudify is used to build the online education cloud. We extend OpenStack's functionalities in order to deal with the challenges.

\section{Architecture of online education cloud}

We use open source cloud platform OpenStack to build the online education cloud. Cloudify [9], an open source framework, is integrated into the OpenStack environment for deploying, managing and scaling our applications. In the network college of East China University of Science and Technology, the online education cloud is designed to provide more than 30,000 students with hundreds of E-learning courseware. As OpenStack continues to gain traction in the customer, partner and vendor worlds, we decide to build and move our online education platform to the OpenStack cloud. Cloudify is an open source framework for deploying, managing and scaling applications on a cloud environment. It is a platform that is designed to support any application regardless of the application stack, can be deployed on any IaaS cloud, and provides full control over the underlying infrastructure to its users. This allows existing applications running on private servers to be directly migrated to the cloud with no modification to code. Vendor lock-in can be avoided due to this flexibility and high level of customizability.

The cloud architecture that we propose is shown in Fig. 1. The architecture is divided into several functional layers. The layered functionalities of the cloud are described as follows.

\section{A. Access layer}

The Access layer offers a unified Web portal into the cloud platform, where online education applications are integrated.

B. Application service layer

The application service layer includes the management module and various kinds of E-learning courseware. The management module reflects basic management functions, including curricula and teaching services, etc. The E-learning courseware contains various online courses which provide students with self-learning, course choosing, homework upload, etc.

C. Supporting layer

The supporting layer is mainly responsible for the management of the cloud platform, including elastic server provisioning which is implemented by Cloudify, resource management, resource scheduling, security, and database management.

D. Virtualization layer 
The virtualization layer is built by OpenStack. It provides services of virtual resources, such as computing, data storage and network communication. The layer is established on physical hardware, which hides the complexity and dynamics of the infrastructure. It implements the management of underlying hardware and gathers dispersed virtual resources into virtual resource pools.

E. Infrastructure layer

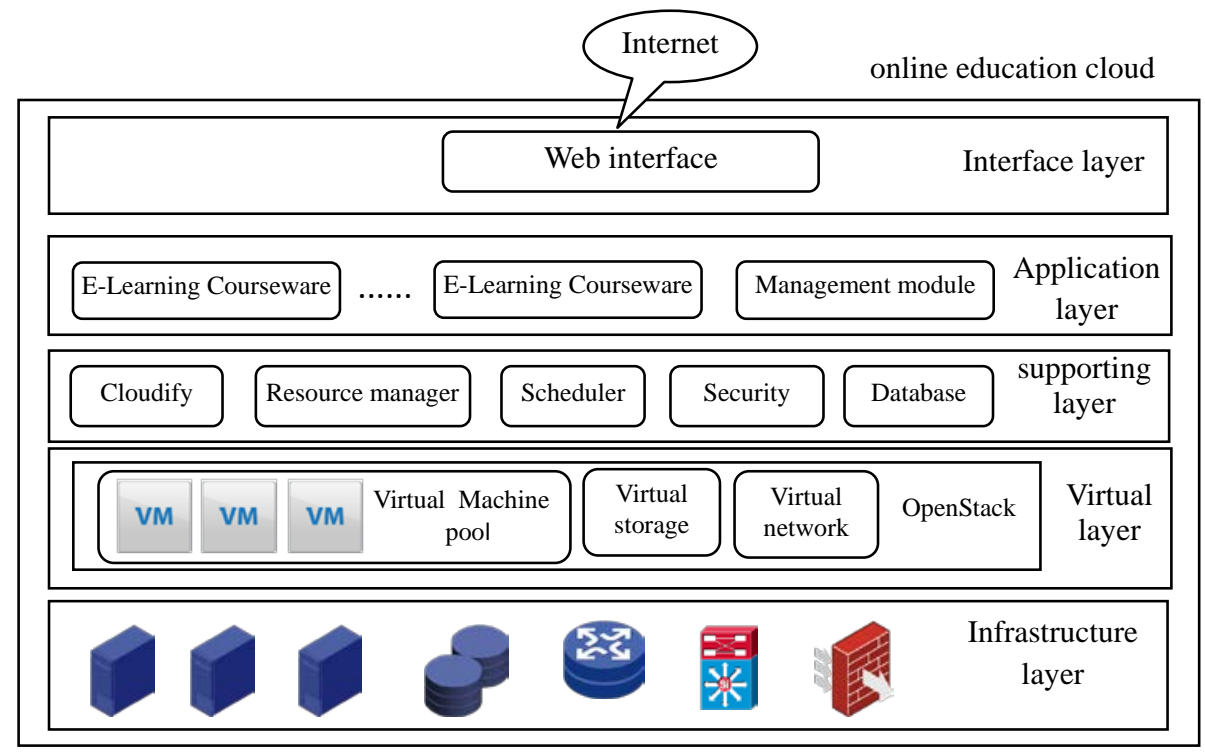

Fig. 1: Architecture of online education cloud

The infrastructure layer provides the infrastructure building the cloud, including physical servers, network equipments and storage devices, etc.

\section{Elastic scheduling strategy of education cloud}

The online education cloud needs to deal with a key issue: the number of online students may change at different times. Our cloud platform needs an automatic scaling ability to ensure services' performance and recycle excess resources according to loads of services. We integrate Cloudify into OpenStack to tackle these issues.

GigaSpaces' Cloudify [9] is an open source PaaS platform. It can conveniently implement an application's scalability without any programming of the corresponding platform. So we integrate Cloudify into OpenStack to make it with the elastic scaling ability of services. When the workload of a service is over the upper threshold, the platform automatically deploy additional instances to share the workload. While the workload decreases below the lower threshold, it will terminate redundant instances to recycle the resources. The proposed scaling architecture is shown in Fig. 2.

In Fig. 2, The Cloudify Cli provides the client environment based on console, and the WebUI provides the user management interface based on Web. The Recipes are configuration files for applications and services. By setting them, new instances will be created in case the users or network throughput of application instances are above the upper threshold, while the loads of instances are lower, part of instances will be destroyed to save resources. The Cloud Controller is the control center that deploys applications and manages automatic scaling. The Cloud Driver is the abstraction layer which provides the cloud infrastructure interface. Cloudify accesses OpenStack through the Cloud Driver to start virtual machines and upload script files and Cloudify components. 


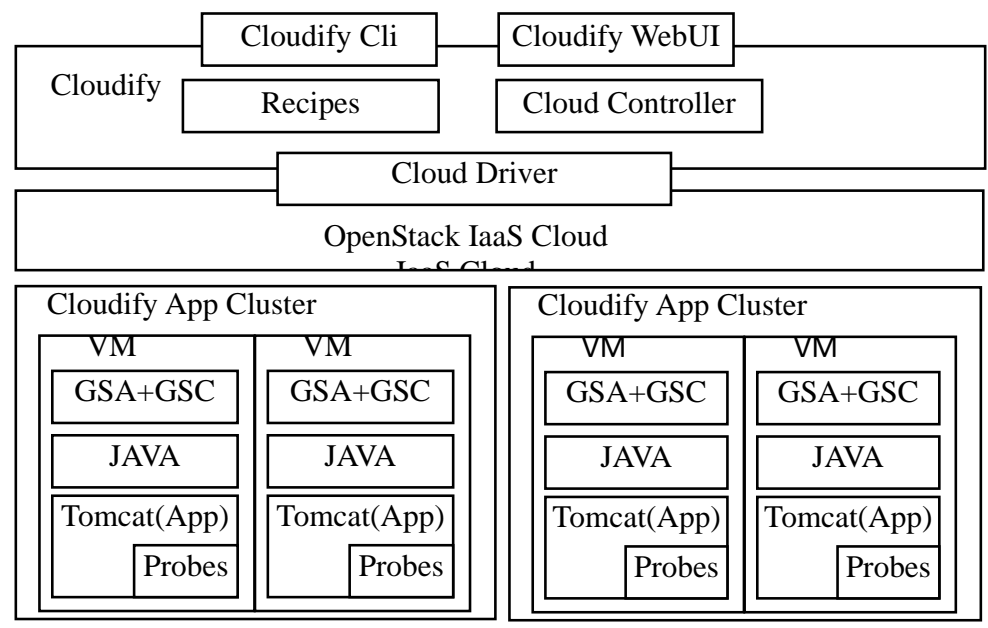

Fig. 2: The architecture of elastic cloud

Scaling cloud services is triggered by the monitor data from cloud agents in virtual machines. When an application provides its service, an agent process of Cloudify continues to monitor the health state of the virtual machine. Once it is down or its load is over the upper threshold, the Cloud Controller will call IaaS API to create a new virtual machine via the Cloud Driver according to the recipe configuration of the application. The Cloud Controller deploys an agent to the new one and makes it work as a new compute node monitored by the agent. The application will be started by the agent to share the load. Similarly, The Cloud Controller will call the cloud driver to recycle excess virtual machines when the load of services is below the lower threshold.

\section{Evaluation}

The response time, which refers to duration time between sending a request to a server and receiving the response from the server, is an important measure of any Web application. Our experiments tested the response times at different concurrency. We compared the response times of a physical server, the original OpenStack cloud and the cloud integrating with Cloudify to evaluate their quality of service.

The experiments used five Dell R320 servers with Xeon 2.4 GHz CPU and 32GB memory. They all run Ubuntu server 14.04 operating system. The OpenStack platform was built by four hosts. One of these hosts is the cloud controller node and three other hosts are compute nodes. Cloudify 3.1 is provisioned on the OpenStack environment. A Web application is deployed in three virtual machines on the OpenStack platform and the same Web application is deployed on a physical server. A Web page with 40k Bytes size is used to test response times. The response times at different concurrency are show in Fig. 3.

Fig. 3 illustrates that when the number of concurrent users is less than 300, the physical server, the original OpenStack, and the improved cloud with Cloudify have almost the same response times. However, when the number of concurrent users is over 300, the response time of the physical machine increases dramatically, the response times of the original OpenStack and the improved platform only increase steadily. This is because two cloud platforms automatically instantiates more instances of the application to share the workload. However, when concurrent users are over 1000, the response time of the improved cloud with Cloudify is gently decreasing. The reason is that the platform automatically deploys more virtual machine instances to share the workload. 


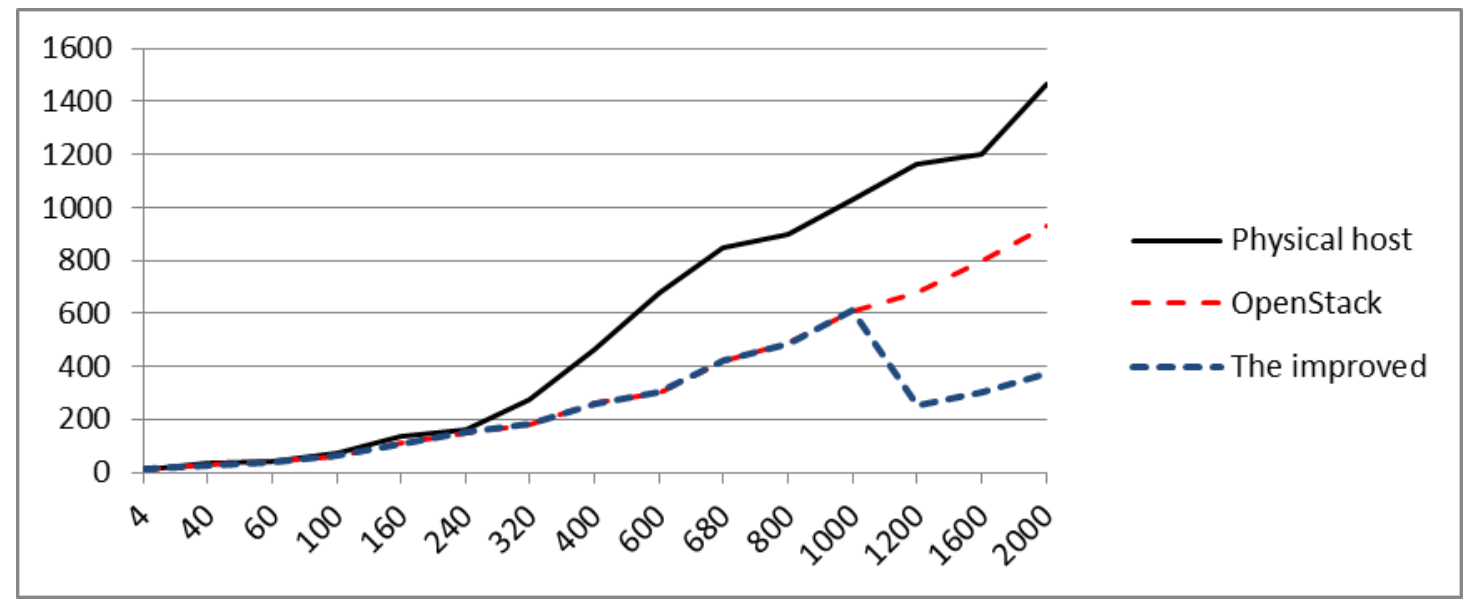

Fig. 3: Performance contrast of online education cloud platform

\section{Conclusion}

In this paper, we have presented a cloud-based online education solution to share cloud resources for various E-learning applications. We discussed an automatic scaling method to optimize the workload management of cloud services. The performance of the services can be ensured by expanding their instance provisioning. Experimental results show that the average response time and load capacity on our education cloud platform are better than ordinary servers and the original OpenStack, so better user experiences can be provided. In the future, we are going to study an innovative scheduling algorithm for balancing resource load, which enables the limited resources of the private education cloud to effectively meet the resource needs of large-scale E-learning.

\section{References}

[1] P. Mell and T. Grance, “The NIST definition of cloud computing," National Institute of Standards and Technology, Special Publication 800-146, 2012.

[2] OpenStack: The Open Source Cloud Operating System [EB/OL]. http://www.openstack.or g/software/, 2014.

[3] C. Ivica, J. Riley, and C. Shubert, "StarHPC: Teaching parallel programming within elas tic compute cloud," in Information Technology Interfaces, 2009. ITI '09. Proceedings of the ITI 2009 31st International Conference on, 2009, pp. 353-356.

[4] CISCO, “Cisco networking academy,” [retrieved: Nov, 2013]. [Online].

[5] S.K. Zhao, L. Li, X, Ling. Architecture and scheduling scheme design of TsinghuaClou d based on OpenStack[J]. Journal of Computer Applications, 2013, 33(12): 3335-3338, 3 349.

[6] C. Pei, Y. C. Wu, Z.Q. Liu, Y.B. Wang.Self-elasticity cloud platform based on OpenSta ck and Cloudify[J]. Journal of Computer Applications, 2014, 34(6): 1582-1586, 1594.

[7] Jing Li, Research and Implementation of Resource Sharing in Distance Education Based on Cloud Computing [D]. Master thesis of Xidian University. 2013.

[8] Ma Zhichao, Research and Optimize of OpenStack of OpenStack Scheduling Algorithm [D]. Master thesis of zhejiang University. 2014.

[9] Cloudify Documentation (Cloudify 3.1) [EB/OL]. [2014-12-17]. http:// www.cloudifysourc e.org. 